\title{
Are Major Basic Protein, Eotaxin-3 and Leukotriene Enzyme Staining Useful for Diagnosing Eosinophilic Esophagitis?
}

\author{
(Am J Gastroenterol 2012;107:1503-1511)
}

\section{Jie-Hyun Kim}

Department of Internal Medicine, Institute of Gastroenterology, Gangnam Severance Hospital, Yonsei University College of Medicine, Seoul, Korea

\section{Summary}

The diagnosis of eosinophilic esophagitis $(\mathrm{EoE})$ is based on the clinical symptoms of esophageal dysfunction and histopathological findings of esophageal eosinophilia. ${ }^{1,2}$ In fact, the clinical, endoscopic, and histopathological characteristics of EoE and gastroesophageal reflux disease (GERD) overlap substantially, and differentiating the two can be difficult. ${ }^{1}$ Recently, Dellon et al ${ }^{1}$ suggested that tissue biomarkers of eosinophil activation or inflammation were useful for diagnosing EoE, especially for differentiating it from GERD. ${ }^{1}$ They performed immunohistochemistry for major basic protein (MBP), eotaxin-3, leukotriene A4 hydrolase (LTA4H) and leukotriene $\mathrm{C} 4$ synthase (LTC4S) in esophageal biopsy tissues from EoE and GERD patients and demonstrated that patients with EoE had much higher levels of MBP and eotaxin-3 in the esophageal epithelium than did GERD patients, while there were no differences in $\mathrm{LTA} 4 \mathrm{H}$ and LTC4S. ${ }^{1}$ They evaluated the diagnostic utility of MBP and eotaxin-3 using receiver operating characteristic curves, and found that the combination of MBP and eotaxin-3 staining and the eosinophil numbers nearly completely predicted EoE status without considering other clinical, endoscopic or histopathological characteristics. ${ }^{1}$

The authors concluded that staining for MBP and eotaxin-3 might improve the current EoE diagnostic standards by adding a measure of eosinophil activity, especially for differentiating it from GERD. ${ }^{1}$

\section{Comment}

The diagnosis of EoE is based on clinical symptoms and endoscopic and histopathological findings. That is, suspicion based on clinical symptoms and endoscopy is important for determining when to perform an esophageal biopsy to diagnose EoE. However, esophageal eosinophilia, presumably the histopathological hallmark of EoE, is frequently seen in esophageal biopsies in GERD. $^{3-5}$ Therefore, it might be important to find other biomarkers for differentiating EoE from GERD.

The pathogenesis of EoE involves an immunological re-

Received: November 28, 2012 Revised: December 22, 2012 Accepted: December 25, 2012

(c) This is an Open Access article distributed under the terms of the Creative Commons Attribution Non-Commercial License (http://creativecommons. org/licenses/by-nc/3.0) which permits unrestricted non-commercial use, distribution, and reproduction in any medium, provided the original work is properly cited.

*Correspondence: Jie-Hyun Kim, MD, PhD

Department of Internal Medicine, Institute of Gastroenterology, Gangnam Severance Hospital, Yonsei University College of Medicine, 211, Eonju-ro, Gangnam-gu, Seoul 135-720, Korea

Tel: +82-2-2019-3505, Fax: +82-2-3463-3882, E-mail: otilia94@yuhs.ac

Financial support: None.

Conflicts of interest: None. 
sponse to allergen exposure, such as Th2 response. This stimulates esophageal epithelial cells to produce eotaxin-3, which leads to eosinophil activation and the release of intracellular granules, including $\mathrm{MBP}^{6,7}$ Other allergic proinflammatory mediators are involved, including the enzymes LTA4H and LTC4S. Therefore, these might be useful biomarkers for diagnosing $\mathrm{EoE}$ and differentiating it from GERD, as the authors mentioned. ${ }^{1}$ Pathophysiologically, the expression of MBP, eotaxin-3, and allergic proinflammatory mediators is upregulated in EoE. ${ }^{10,11}$ However, in this study, the correlation between eotaxin-3 density and eosinophil count was weaker $(r=0.25)$ than between MBP and eosinophil count even after considering for the different age distribution in the case and control groups unlike the previous studies. It may be necessary to investigate further why eotaxin-3 density did not correlate strongly with eosinophil count different from MBP.

It is important to determine whether upregulated expressions of MBP, eotaxin-3 and allergic proinflammatory mediators are clinically useful for determining the diagnosis or prognosis. Therefore, this study is valuable for investigating the diagnostic utility of these markers, focusing, in particular, on the differential diagnosis of GERD. Furthermore, this is a notable study which examined the diagnostic utility of MBP and eotaxin-3 in EoE with receiver operating characteristic analysis. Quantitative immunohistochemistry is a more available method to be used in the clinical field than other protein or mRNA measurements.

However, the diagnostic markers might be more useful for differentiating EoE from GERD, than for the diagnosis of $\mathrm{E} o \mathrm{E}$ itself, as the authors mentioned, because there are definite diagnostic criteria for EoE, such as the eosinophil count. Consequently, it is important to define a study population that is clinically ambiguous in terms of EoE and GERD. In this study, the authors analyzed the following subgroups: (1) a GERD group with erosive esophagitis only, (2) a GERD group with $\geq 15$ eosinophils per high power field on esophageal biopsy, and (3) an EoE group with $<100$ eosinophils per high power field on esophageal biopsy. No data for analyses involving more ambiguous subgroups were shown in the manuscript. However, it would have been better to show the data for such subgroup analyses because the results might have more relevance for clinically ambiguous groups in terms of GERD and EoE.

Furthermore, an analysis of more ambiguous subgroups might be helpful for differentiation between EoE and GERD. For example, a comparison between EoE and GERD both with normal endoscopic findings, or between EoE and GERD both with erosive esophagitis, or between proton pump inhibitor responsive EoE and GERD; that is, subgroups that are difficult to differentiate clinically or endoscopically. We hoped that this study would have suggested specific criteria for using MBP or eotaxin-3 to differentiate EoE from GERD. Nevertheless, this study presents valuable data on diagnostic biomarkers of EoE.

\section{References}

1. Dellon ES, Chen X, Miller CR, Woosley JT, Shaheen NJ. Diagnostic utility of major basic protein, eotaxin-3, and leukotriene enzyme staining in eosinophilic esophagitis. Am J Gastroenterol 2012;107:1503-1511.

2. Furuta GT, Liacouras CA, Collins MH, et al. Eosinophilic esophagitis in children and adults: a systematic review and consensus recommendations for diagnosis and treatment. Gastroenterology 2007; 133:1342-1363

3. Ngo P, Furuta GT, Antonioli DA, Fox VL. Eosinophils in the esophagus - peptic or allergic eosinophilic esophagitis? Case series of three patients with esophageal eosinophilia. Am J Gastroenterol 2006;101:1666-1670.

4. Rodrigo S, Abboud G, Oh D, et al. High intraepithelial eosinophil counts in esophageal squamous epithelium are not specific for eosinophilic esophagitis in adults. Am J Gastroenterol 2008;103:435-442.

5. Dellon ES, Farrell TM, Bozymski EM, Shaheen NJ. Diagnosis of eosinophilic esophagitis after fundoplication for 'refractory reflux': implications for preoperative evaluation. Dis Esophagus 2010;23: 191-195.

6. Rothenberg ME. Biology and treatment of eosinophilic esophagitis. Gastroenterology 2009;137:1238-1249.

7. Rothenberg ME, Hogan SP. The eosinophil. Annu Rev Immunol 2006;24:147-174.

8. Gupta SK, Peters-Golden M, Fitzgerald JF, et al. Cysteinyl leukotriene levels in esophageal mucosal biopsies of children with eosinophilic inflammation: are they all the same? Am J Gastroenterol 2006; 101:1125-1128.

9. Attwood SE, Lewis CJ, Bronder CS, Morris CD, Armstrong GR, Whittam J. Eosinophilic oesophagitis: a novel treatment using Montelukast. Gut 2003;52:181-185.

10. Kephart GM, Alexander JA, Arora AS, et al. Marked deposition of eosinophil-derived neurotoxin in adult patients with eosinophilic esophagitis. Am J Gastroenterol 2010;105:298-307.

11. Blanchard C, Wang N, Stringer KF, et al. Eotaxin-3 and a uniquely conserved gene-expression profile in eosinophilic esophagitis. J Clin Invest 2006;116:536-547. 